\title{
Effects of Music Therapy on Well-Being of People with Alzheimer's Disease and Related Dementia
}

\author{
Lingzi Zhang ${ }^{1}$
}

${ }^{1}$ The Hockaday School, Dallas, TX, USA

\section{Introduction}

For centuries, music has been an inherent part of our culture and life. While many people think of music as a practice of enjoyment, Sacks $(2008,386)$ states that music is "...a necessity" for patients with dementia, because it "...can have a power beyond anything else to restore them to themselves." Alzheimer's Disease (AD) and related dementia causes irreversible damage to the brain, and since there is no cure, it is not possible to elicit memories that are already lost. Therefore, Sacks $(2008,381)$ suggests that music can evoke emotional responses by giving patients with AD and related dementia access to the remaining memory of music-related experiences. This connection between music and memory makes music therapy (MT) a feasible treatment for patients with AD and related dementia. As studies show, MT promotes the mental health and well-being of these patients by reducing psychological symptoms and the amount of psychotropic medication prescribed (Ridder et al. 2013); thus, MT improves the patients' quality of life. MT also has some physiological benefits, including a decrease in blood pressure and increase of appetite and food intake among patients (Takahashi and Matsushita 2006).

The unique relationship between people with $\mathrm{AD}$ and related dementia and music inspired me to write this article on MT's potential to promote the well-being of patients with AD and related dementia in nursing homes, particularly in the United States. Since the total number of people affected by AD and related dementia in the US is over 5 million and that number is expected to increase three-fold by 2060 as the population ages (Center for Disease Control and Prevention 2018; Vespa 2019), it is important that we employ more effective treatments that can relieve some symptoms of $\mathrm{AD}$ and related dementia to lessen the burden on families.

\section{Background}

\section{Introduction to Music Therapy}

Music therapy (MT) is a form of clinical treatment led by a professional who has specific training in both the scientific and musical fields and uses music to accomplish individualized, often therapeutic, goals (American Music Therapy Association, n.d.; Seibert 2016). There are two main types of MT intervention: passive and active. Passive intervention involves listening to live or recorded music - the patient does not directly engage with the music or the music therapist (Prakash, n.d.; American Music Therapy Association, n.d.). However, music listening is only considered part of clinical MT when the music therapist intentionally selects pieces and songs for listening based on the patient's experience and preference (Prakash, n.d.). On the other hand, active intervention, a technique that clinical music therapists most often employ, involves active interaction by the patient with music and the music therapist in many ways, such as singing songs and playing musical instruments together (Prakash, n.d.; American Music Therapy Association, n.d.).

A MT session is highly individualized. Before a session, the music therapist will investigate the patients' preferences, life experience, and severity of their diseases. This will inform how the music therapist will approach the session (e.g. what instrument and genre of music to use) in order to maximize the benefits for the patient (American Music Therapy Association, n.d.). In a typical MT session, the music therapist plays or sings specific pieces or songs 
that relate to the patient's personal experience, usually music with which the patient is familiar from a younger age, to evoke the patient's memories and emotions, induce a reaction from the patient, and thus encourage them to interact with other people (Sacks 2008, 381), such as their family members and their caregivers.

A MT session is not limited to music-making by the therapist only, however. The music therapist can interact with the patient by improvising or composing together, giving instructions on how to play certain musical instruments, and potentially providing music-related movement opportunities such as finger exercises (American Music Therapy Association, n.d.; Takahashi and Matsushita 2006).

\section{Dementia and the Main Symptoms}

MT is often used to treat patients with age-related, progressive neurodegenerative diseases of which the main symptom is dementia. The most common cause of dementia is Alzheimer's Disease (AD), which consists of $60 \%-80 \%$ of the cases (Alzheimer's Association, n.d.). Diseases like AD can affect the patient both cognitively - shown by symptoms such as loss of memory and difficulty communicating, reasoning, solving problems, etc. - and psychologically shown by symptoms such as depression, agitation, and hallucination (Mayo Clinic 2019). These diseases are progressive, meaning that the severity of the dementia symptoms worsens over time. Since there is currently no cure to these diseases, the medicine and treatment available only slows down the development of the symptoms (Alzheimer's Association, n.d.). Meanwhile, the long span of time (four to eight years on average) that AD affects a person increases families' financial and emotional burden (Alzheimer's Association, n.d.). With an aging population in the US, the number of people with AD and related dementia likely will continue to increase (Center for Disease Control and Prevention 2018), thus posing a challenge to American families and presenting the need for more effective treatments.

\section{Music, Memory, and Dementia}

\section{Music and the Brain}

Although music plays a significant role in many people's lives, the term "music" remains vague. In the context of this article, "music" refers to a combination of organized vocal, instrumental, and synthesized sounds that follow certain rules. Moreover, music is an umbrella term covering many styles, such as classical, pop, rock, and jazz, but there is not one specific style that music therapists most commonly use, for they aim to address an individual's unique experience related to music (American Music Therapy Association, n.d.). Thus, different sessions may require different types of music.

Music is a complex notion, because even a simple melody has key elements such as pitch, tempo, rhythm, and harmony. The intricacy of musical works, therefore, makes processing of music a "whole-brain" activity that requires various parts of the human brain to work together (Warren 2008). The brain first processes music in the ascending auditory pathway, from the cochlea to primary auditory cortex (Warren 2008), in the same way that we normally perceive and process non-musical sounds, However, unlike non-musical sounds, music also requires processing in other parts of the brain (Fig. 2). For instance, the element of pitch alone is processed in three different parts of the brain - the anterior superior temporal gyrus, the lateral Heschl's gyrus, and the planum temporale - while emotions related to the music are processed in the limbic circuit, a fundamental component of the limbic system (Warren 2008; Elgendy and Bell, n.d.). The limbic system is a structure in the brain that regulates the biochemical and autonomic responses to emotions as well as the expression of emotions through movement (Koelsch 2014). Processing music arouses activity in the limbic system, making MT possible. 


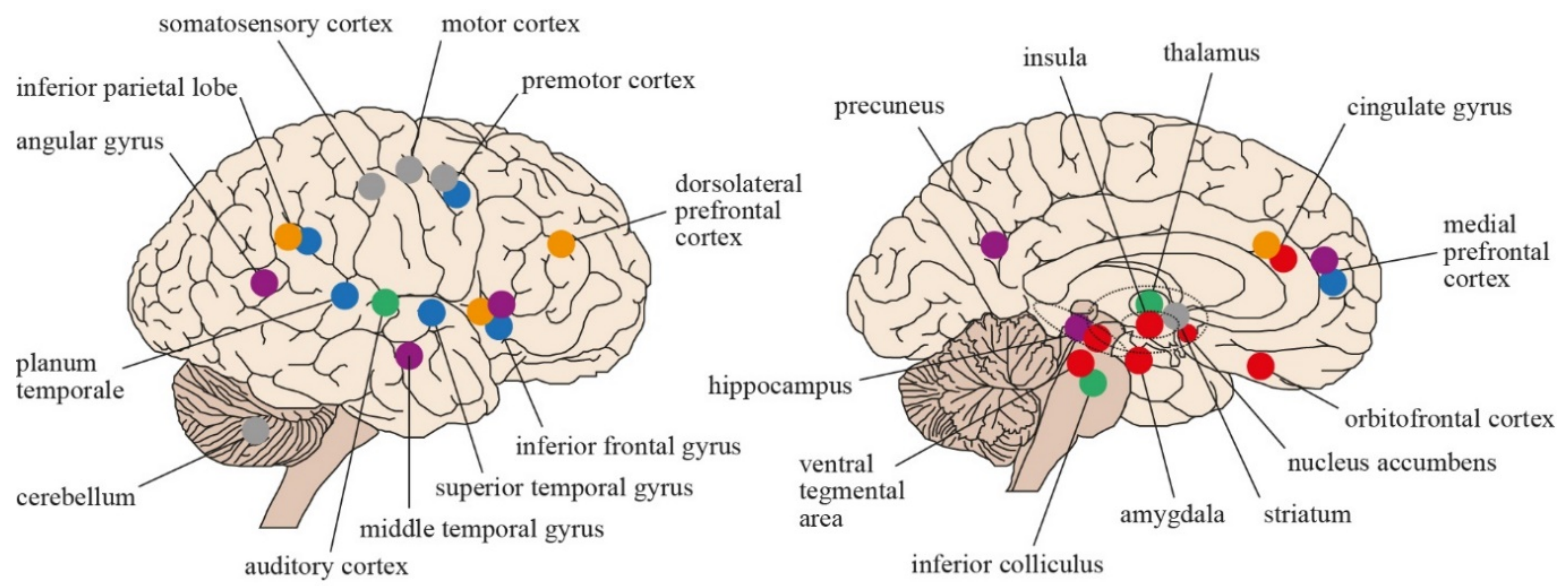

Perceiving the basic acoustic features of music (e.g., frequency, duration, loudness)

Perceiving higher-order musical features (e.g., harmony, intervals, rhythm)

Focusing and keeping track of music in time (attention, working memory)

Recognizing music and recalling associated memories (episodic memory)

Playing, singing and moving to the beat of music (motor functions)

Music-evoked emotions and experiencing pleasure and reward

Figure 1. Key Brain Areas Associated with the Processing of Music.

\section{Music and Memory}

As shown by figure 3, there are three main types of memory: sensory, short-term, and long-term memory (Boundless Psychology, n.d.). Under long-term memory, there are explicit and implicit memories (Boundless Psychology, n.d.). Explicit memory is further split into two categories: semantic and episodic memory, which are main components of autobiographical memory (Holland and Kensinger 2010; Boundless Psychology, n.d.). More specifically, semantic memory stores facts about one's self and the surrounding world (e.g. the name of a specific person or country, the time that World War II started) (Holland and Kensinger 2010). Meanwhile, episodic memory stores events and personal experiences (e.g. a wedding, a vacation, how one felt during said event or experience) and requires the retrieval of information about certain events in the past (Holland and Kensinger 2010; Wheeler, Struss, and Tulving 1997). Since music can evoke the patient's memory of experiences (Fig. 2), the use of MT mainly targets the patient's episodic memory (Fusar-Poli et al. 2017; Baird and Samson 2009; Särkämö et al. 2012). 


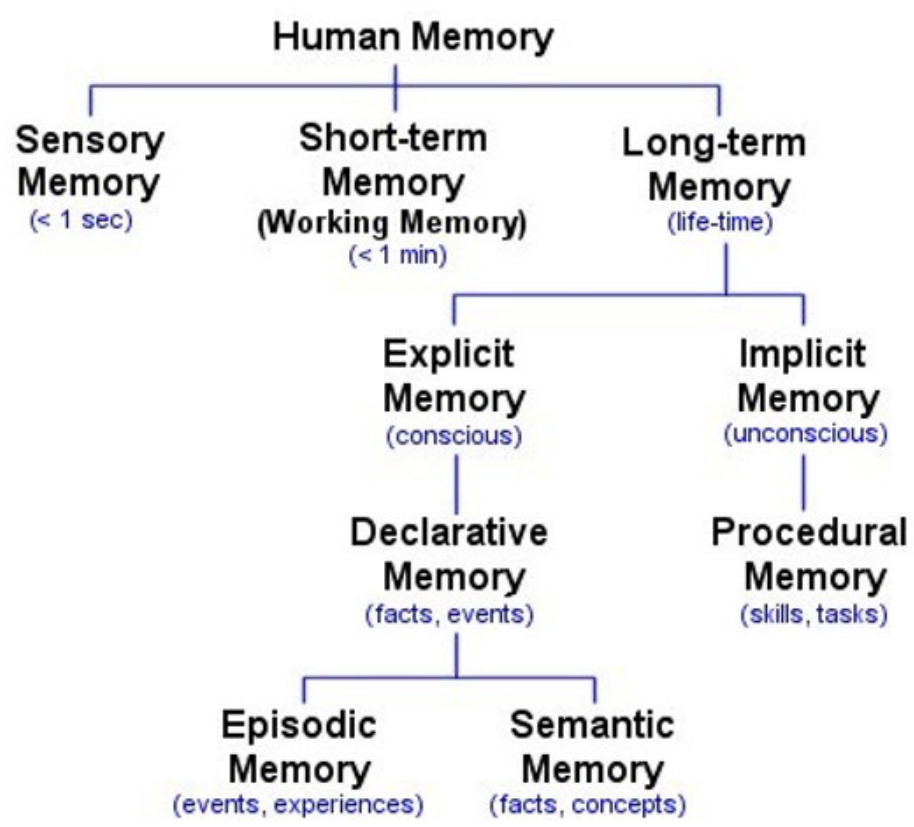

Figure 2. Different Types of Memory.

Music can evoke autobiographical memories related to certain songs and pieces; thus, music plays a significant role in the formation of the view of one's own self (Jäncke 2008). Interestingly, the brain areas for memory of music and musical experiences are one of the last to deteriorate among patients with AD and related dementia (Fusar-Poli et al. 2017; Jacobsen et al. 2015). As a result, patients are able to feel the emotions about and react to certain music until the end stage of dementia (Fusar-Poli et al. 2017). It is this characteristic of musical memory that makes MT a suitable treatment, especially for patients with neurodegenerative diseases like AD.

\section{Different Types of Dementia}

Alzheimer's Disease (AD) is not the only progressive disease that causes dementia and interferes with a person's memory; some other examples are Lewy Body Dementia, Parkinson's Disease, and Posterior Cortical Atrophy, etc. (Alzheimer's Association n.d.). Dementia can also be caused by nondisjunction, unequal separation of chromosomes during meiosis (e.g. Down Syndrome) and other genetic factors (Alzheimer's Association n.d.). This paper mainly examines the effect of MT on patients with AD and related dementia due to the abundance of studies done in this area.

\section{Methods}

Partly due to the Covid-19 pandemic, I was not able to interview caregivers and collect data on the state of well-being in relation to music therapy of some patients at nursing homes; therefore, I relied on the published literature for information for analysis. Keywords such as "Alzheimer's Disease," "dementia," "memory," and "music therapy and dementia" were searched on Google, Google Scholar, National Library of Medicine, and Pro-Quest. In particular, I drew material from the Journal of Music Therapy and obtained general information about dementia and MT from the American Music Therapy Association (AMTA) and the Center for Disease Control and Prevention (CDC). In addition, I consulted the book Musicophilia: Tales of Music and the Brain by Oliver Sacks and watched a TEDx Talk given by a professional music therapist, Erin Seibert, to further my understanding of MT and dementia. Then, I grouped into several categories to be further discussed. By close reading the literature and analyzing the researchers' observations, 
I reached provisional conclusions that MT has a positive effect on the patients' well-being as well as health and should be employed as a supplementary treatment.

\section{Discussion}

The evidence I discuss in this section are from trials conducted by other researchers with patients at the dementia units in nursing homes. These patients have an average age of at least 78. The findings of this article shows that MT can relieve many of the psychological symptoms of dementia and enhance these patients' quality of life. There is also evidence of potential positive health effects for patients with $\mathrm{AD}$ and related dementia, although no beneficial outcome of MT on the cognitive function of patients was found.

\section{Beneficial Effects of MT}

\section{Psychological Effects on Well-Being}

As briefly mentioned in the introduction and background sections, for patients diagnosed with dementia, the use of MT aims to promote their well-being by reducing the psychological symptoms of dementia - helping the patient reobtain a sense of self and facilitating emotional connections with others around them (American Music Therapy Association, n.d.; Sacks 2008, 372-373). Although MT is a type of clinical treatment, it is considered nonpharmacological (i.e. does not involve any medication), which means it can decrease the patients' risk of exposure to harmful side effects of medication from other treatments (American Music Therapy Association, n.d.).

An experiment by Ray and Götell (2018) showed that MT conducted by credentialed music therapists and trained certified nursing assistants significantly decreased depression symptoms among patients with moderate dementia. By extension, MT can possibly evoke more positive emotions and improve the patients' mental health. Furthermore, Ridder et al. (2013) found in their study that MT reduced agitation disruptiveness and aggression among patients with $\mathrm{AD}$ and related dementia and was especially effective when techniques of active MT were employed. As a result, the patients treated with MT were prescribed fewer psychotropic medications meant to prevent disruptive behaviors than patients not treated with MT. Psychotropic medication can have harmful side-effects, such as hallucination (Ridder et al. 2013), which can cause unsettling disruptions to the patients' perception of their environment. In other words, MT decreases agitation disruptiveness and also indirectly reduces hallucination caused by medication, which improves the patients' sense of well-being by providing more stability.

Discussed in the same study, Ridder et al. (2013) mention that MT can potentially reduce aggression and increase patients' level of compliance and the degree of reciprocity between the patient and the caregiver. Such effects of MT can increase the amount and duration of the patients' engagement with others, specifically in a "one-to-one socializing" situation (Ridder et al. 2013; Cohen-Mansfield et al., 2011). Even when the patients' verbal communication abilities deteriorate, MT allows the patients to establish contact with others through music and emotions (Brotons and Koger, 2000). This effect can potentially lessen the burden of families because the patient would be more willing to cooperate for treatment and communication.

\section{Physiological Effects on Well-Being}

Besides psychological effects, MT is also found to be effective in promoting the general health of patients with AD and related dementia. Kumar et al. (1999) found that blood melatonin levels increased significantly among the patients treated with MT. High blood melatonin levels are tied to improved sleep quality, which allows the body to better repair and rehabilitate. Besides improving patients' sleep quality, MT has been shown to increase patients' appetite and food intake (Takahashi and Matsushita 2006), which also shows that MT promotes a healthy state of being.

Apart from general effects on the patients' well-being, some researchers have obtained results demonstrating that MT is effective in enhancing the patients' immune system and preventing some serious diseases. After studying 
19 AD patients, Hasegawa et al. (2001) found that the percentage and activity of natural killer cells, part of the human immune system, increased during MT sessions, which can possibly strengthen these patients' immune systems over time and make patients less susceptible to many chronic diseases (Takahashi and Matsushita 2006).

Similarly, experiments have shown that patients treated with MT have lower systolic blood pressure (Takahashi and Matsushita 2006), which suggests that MT is effective in decreasing the patients' risk of cardiac and cerebral diseases, since these diseases are usually associated with high systolic blood pressure. Aforementioned studies indicate that MT may lower the patients' susceptibility to serious chronic diseases while having almost no harmful side effects. Therefore, MT should be considered an efficacious way to promote the health of patients and lessen the burden of caregivers and patients' families.

\section{Limitations of MT}

However, researchers clarify that MT has not been found to be effective in improving cognitive functions, for MT cannot reverse effects of dementia or elicit memory that used to exist in the brain parts that are now damaged and dysfunctional. For instance, Fusar-Poli et al. (2018) stated that similar to many other researchers in the field, they did not find any positive effects of MT on patients' global cognition and cognitive functions such as memory, language, etc., implying that MT is mainly a treatment promoting the well-being of the patients, not one that can eliminate symptoms of AD and related dementia such as loss of memory. Hence, MT cannot replace medical treatment, but it can be incorporated as part of the holistic treatment to improve the patients' quality of life. As Sacks (2008, 378) said, "singing cannot be used as a sort of back door to explicit memory, still the act of singing is important in itself," because all the effects of MT that promote well-being mentioned in the paragraphs above can largely benefit patients with AD and related dementia, leading them to a better life.

Some other limitations are related to the practicality of the application of MT. One limitation is that there are not enough board-certified music therapists to meet the demand, keeping the patients from accessing this effective, nonpharmacological treatment. In the US only, there are only 6500 board-certified music therapists (Taub 2016), while the number of people diagnosed with $\mathrm{AD}$ and related dementia is around 800 times more, at 5 million (Center for Disease Control and Prevention 2018). Obviously, one music therapist could not treat 800 patients on a regular basis, meaning that many patients with $\mathrm{AD}$ and related dementia have very limited any access to clinical MT, if at all. Moreover, the rate for music therapists can be prohibitive for many families, at $\$ 60$ to $\$ 100$ per hour (Taub 2016). Nevertheless, the benefits of MT have been proved to be significant, so more resources should be allocated to encourage people to become music therapists and meet the increasing demand for more effective treatments.

\section{Recommended Approach to MT}

To apply MT more strategically, researchers have proposed different approaches to MT for patients with varying severity of dementia. Although Takahashi and Matsushita (2006) pointed out that active intervention of MT yields more effective results than passive intervention, active intervention is not suitable for all patients, for patients in the later stages of dementia may not be able to actively engage in the MT process. Therefore, it would be most reasonable that music therapists use active intervention for patients in the earlier stages of dementia and passive intervention for patients who are no longer mentally or physically able to engage in MT sessions (Takahashi and Matsushita 2006).

\section{Conclusion}

Through examining studies conducted by other researchers, I have found various beneficial effects of MT on people with $\mathrm{AD}$ and related dementia, ranging from relieving psychological symptoms of dementia, decreasing the risk for some chronic diseases, and promoting the general well-being of the patients. MT can also help these patients find a 
sense of self and bond with their families and others around them, relieving some of the emotional burden on families and caregivers. Since active MT has been proved to yield more beneficial results than passive MT, music therapists should apply active MT when possible and appropriate. However, MT has its limitations: It is not a panacea, for it cannot reverse the effects of damaged brain parts and has not been found to improve the patients' cognitive function, such as memory and ability to solve problems. What MT can do is to improve the mental state of the patients and relieve the pain and discomfort they have to go through from drug therapy. All medicines have side effects, and the more medicines the patients take, the more negative side effects to which patients can be exposed. These side effects can seriously worsen the patients' quality of life. Since MT has been found to reduce the amount of medicines prescribed, MT can promote patients' physical and mental health. Therefore, based on the literature review, I conclude that MT should be recommended to patients (in conjunction with other forms of clinical treatment) for its beneficial effects on psychological symptoms and the patients' overall well-being.

\section{Acknowledgements}

I would like to thank Dr. Roger Worthington, an independent researcher based in London, UK, for giving me instructions and support throughout researching about this topic and constructing this paper. This paper and the research would not have been possible without him and I truly appreciate his unique insights.

\section{References}

Alzheimer's Association. n.d. “What Is Alzheimer's Disease?” Accessed May 24, 2020. https://www.alz.org/alzheimers-dementia/what-is-alzheimers.

American Music Therapy Association. n.d. "Frequently Asked Questions.” Accessed May 28, 2020. https://www.musictherapy.org/faq/.

American Music Therapy Association. n.d. "Music Therapy and Alzheimer's Disease.” Accessed May 28, 2020. https://www.musictherapy.org/assets/1/7/MT Alzheimers 2006.pdf.

Baird, Amee, and Séverine Samson. 2009. "Memory for Music in Alzheimer's Disease: Unforgettable?" Neuropsychology Review 19, no. 1 (February 13): 85-101. https://doi.org/10.1007/s11065-009-9085-2.

Body and Mind staff. 2012. "Music Therapy Helps Nursing Home Residents.” PennLive. Updated January 5, 2019. https://www.pennlive.com/bodyandmind/2012/04/the healing power of music the.htm.

Boundless Psychology. n.d. "Introduction to the Process and Types of Memory." Accessed June 8, 2020. https://courses.lumenlearning.com/boundless-psychology/chapter/introduction-to-memory/.

Brotons, M., and S. M. Koger. 2000. "The Impact of Music Therapy on Language Functioning in Dementia." Journal of Music Therapy 37, no. 3 (Fall): 183-95. doi:10.1093/jmt/37.3.183.

Centers for Disease Control and Prevention. 2018. "U.S. Burden of Alzheimer's Disease, Related Dementias to Double by 2060." Published September 20, 2018. https://www.cdc.gov/media/releases/2018/p0920-alzheimers-burdendouble-2060.html. 
Cohen-Mansfield, Jiska, Marcia S. Marx, Laurence S. Freedman, Havi Murad, Natalie G. Regier, Khin Thein, and Maha Dakheel-Ali. 2011. "The Comprehensive Process Model of Engagement." The American Journal of Geriatric Psychiatry 19, no. 10 (October): 859-70. https://doi.org/10.1097/jgp.0b013e318202bf5b.

Elgendy, Azza and Daniel J Bell. n.d. "Papez Circuit: Radiology Reference Article." Radiopaedia Blog RSS. Accessed July 11, 2020. https://radiopaedia.org/articles/papez-circuit-1?lang=us\#: :text=The Papez circuit is a,as the medial limbic circuit.

Fusar-Poli, Laura, Łucja Bieleninik, Natascia Brondino, Xi-Jing Chen, and Christian Gold. 2017. "The Effect of Music Therapy on Cognitive Functions in Patients with Dementia: A Systematic Review and Meta-Analysis." Aging \& Mental Health 22, no. 9 (June): 1103-12. https://doi.org/10.1080/13607863.2017.1348474.

Hasegawa, Yoshiya, Nobuko Kubota, Toshiaki Inagaki, and Nagao Shinagawa. 2001. "Music Therapy Induced Alternations in Natural Killer Cell Count and Function." Nippon Ronen Igakkai Zasshi. Japanese Journal of Geriatrics 38, no. 2 (March): 201-04. doi:10.3143/geriatrics.38.201.

Holland, Alisha C., and Elizabeth A. Kensinger. 2010. "Emotion and Autobiographical Memory." Physics of Life Reviews 7, no. 1 (March): 88-131, https://doi.org/10.1016/j.plrev.2010.01.006.

Jacobsen, Jörn-Henrik, Johannes Stelzer, Thomas Hans Fritz, Gael Chételat, Renaud La Joie, and Robert Turner. 2015. "Why Musical Memory Can Be Preserved in Advanced Alzheimer's Disease." Brain 138, no. 8 (June 03): 2438-450. doi:10.1093/brain/awv135.

Jäncke, Lutz. 2008. "Music, Memory and Emotion.” Journal of Biology 7, no. 6 (August). https://doi.org/10.1186/jbiol82.

Koelsch, Stefan. 2014. "Brain Correlates of Music-Evoked Emotions.” Nature Reviews Neuroscience 15, no. 3 (March): 170-80. https://doi.org/10.1038/nrn3666.

Kumar, A. M., F. Tims, D. G. Cruess, M. J. Mintzer, G. Ironson, D. Loewenstein, R. Cattan, J. B. Fernandez, C. Eisdorfer, M. Kumar. 1999. "Music Therapy Increases Serum Melatonin Levels in Patients with Alzheimer's Disease.” Alternative Therapeutic Health Medicine 5, no. 6 (November): 49-57, https://pubmed.ncbi.nlm.nih.gov/10550905/.

Mayo Clinic. 2019. "Dementia." April 19, 2019. https://www.mayoclinic.org/diseases-conditions/dementia/symptoms-causes/syc-20352013.

O’Kelly, Julian. 2016. "Music Therapy and Neuroscience: Opportunities and Challenges." Voices: A World Forum for Music Therapy 16, no. 2 (2016). https://voices.no/index.php/voices/article/view/2309/2064.

Prakash, Roshni. n.d. "Passive and Active Music Therapy." Voices Together Music Therapy and Autism in Schools. Accessed May 24, 2020. https://sites.duke.edu/voicestogether/series-the-potential-power-of-music-therapy-withinthe-autism-community/passive-and-active-music-therapy/.

Ray, Kendra D., and Eva Götell. 2018. "The Use of Music and Music Therapy in Ameliorating Depression Symptoms and Improving Well-Being in Nursing Home Residents with Dementia." Frontiers in Medicine 5 (October 9). https://doi.org/10.3389/fmed.2018.00287. 
Ridder, Hanne Mette O., Brynjulf Stige, Liv Gunnhild Qvale, and Christian Gold. 2013. "Individual Music Therapy for Agitation in Dementia: An Exploratory Randomized Controlled Trial.” Aging \& Mental Health 17, no. 6 (April 23): 667-78. https://doi.org/10.1080/13607863.2013.790926.

Sacks, Oliver. 2008. Musicophilia: Tales of Music and the Brain. New York: Vintage Books. Kindle.

Särkämö, T., S. Laitinen, M. Tervaniemi, A. Numminen, M. Kurki, and P. Rantanen. 2012. "Music, Emotion, and Dementia: Insight from Neuroscientific and Clinical Research." Music and Medicine 4, no. 3 (2012): 153-62. https://doi.org/10.1177/1943862112445323.

Seibert, Erin. 2016. Why I Want to Change the World with Music Therapy. TEDx Talks. Published January 11, 2016. https://www.youtube.com/watch?v=47-90fPyQa8.

Takahashi, T., and H. Matsushita. 2006. "Long-Term Effects of Music Therapy on Elderly with Moderate/Severe Dementia.” Journal of Music Therapy 43, no. 4 (December): 317-33. https://doi.org/10.1093/jmt/43.4.317.

Taub, Eric. 2016. "Music Therapy Has Industry's Ear.” Seniors Housing Business. Published March 11, 2016. https://seniorshousingbusiness.com/music-therapy-has-industry-s-ear/.

The Human Memory. 2019. "Types of Memory: Different Brain Memories Facts, Jobs." Updated September 27, 2019. https://human-memory.net/types-of-memory/.

Vespa, Jonathan. 2019. "The Graying of America: More Older Adults Than Kids by 2035.” The United States Census Bureau. Updated October 8, 2019. https://www.census.gov/library/stories/2018/03/graying-america.html.

Warren, Jason. 2008. "How Does the Brain Process Music?" Clinical Medicine 8, no. 1 (February): 32-36. https://doi.org/10.7861/clinmedicine.8-1-32.

Wheeler, M.A., D.T. Struss, and E. Tulving. 1997. "Toward a Theory of Episodic Memory: The Frontal Lobes and Autonoetic Consciousness.” Psychological Bulletin 121, no. 3 (May): 331-54. https://doi.org/10.1037/00332909.121.3.331. 